\title{
Detection Method for Counterfeit Currency Based on Bit-Plane Slicing Technique
}

\author{
Mohammad H Alshayeji ${ }^{1 *}$, Mohammad Al-Rousan ${ }^{2}$ and Dunya T. Hassoun ${ }^{3}$ \\ ${ }^{1,2,3}$ Computer Engineering Department, College of Computing Sciences and \\ Engineering, Kuwait University, Kuwait

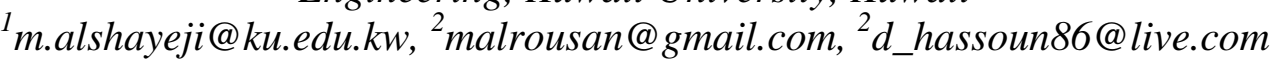

\begin{abstract}
Counterfeiting and forging currencies is a serious threat to any economy. Even though currency exists as a variation of coins, banknotes, and electronic data, many economies remain threatened by counterfeiting which is made possible by the ongoing technological advancements in reprographic equipment available to the general public. Clearly, counterfeit currency detection is not a task that can be neglected. Digital image processing is one of the most common and effective techniques used to distinguish counterfeit banknotes from genuine ones. A new approach is presented in this paper using the bit-plane slicing technique to extract the most significant data from counterfeit banknote images with the application of an edge detector algorithm. The proposed technique consists of decomposing original images of 256 gray levels into their equivalent 8 binary images. This is useful in analyzing the relative importance contributed by each bit of the original image. Higher order bit levels are evaluated for grayscale banknote images with the application of Canny edge detection algorithm. The results are then compared with genuine banknotes and with other existing techniques used for detecting counterfeit notes. Unlike existing research, it was observed that the edges obtained using bit-plane sliced images are more accurate and can be detected faster than obtaining them from the original image without being sliced. The detection of counterfeit currency was also achieved by following the process of using Canny edge detection, image segmentation, and feature extraction.
\end{abstract}

Keywords: Bit-plane slicing, Canny edge detector, Counterfeit banknotes, Currency detection, Feature extraction

\section{Introduction}

The growing menace of counterfeit currency is evident as newspapers and online articles report huge caches of fake currency notes being seized more often [1]. The amount of circulation of counterfeit money can threaten the reliability of state currency at any time, which in return has an impact on the stability and value of currency [2]. Different anti-counterfeit approaches and measures have been developed on currency notes to be able to cope with the act of counterfeiting. Some of the measures available in currency notes include watermarks, security threads, and holograms. However, the constant and rapid improvements in the scanning and printing industries have made it easier for unskilled counterfeiters to reproduce high-quality and close to reality banknotes.

In this paper, image processing is used to manipulate the nature of images in order to enhance pictorial information which can be easily interpreted by humans [3]. The images used and analyzed are digital images. A digital image is an image composed of discrete values, which can be considered as an array of discrete dots with their associated

${ }^{*}$ Corresponding Author 
brightness [3]. These dots are picture elements and are referred to as pixels. A grayscale image is the preferred format for image processing in this paper. When the acquired images are of Red-Green-Blue (RGB) color, they can be decomposed and processed as three separate grayscale images for simplicity.

When processing digital images, the images have to go through several phases before beneficial information can be extracted from the image. Images of scanned currency notes used in this paper will undergo the following phases: image acquisition, pre-processing an image, bit-plane slicing, edge detection, image segmentation, and feature extraction. Enhancing images may not always be the answer for image analysis, depending on the application. This can be resolved using bit-plane slicing where relative information can be extracted from each bit-plane [4].

In this paper, Section 2 discusses existing work related to currency recognition and the detection of counterfeit currency. Section 3 and 4 briefly explain counterfeit currency and digital image processing, while Section 5 describes the proposed method using bit-plane slicing technique. Section 6 explains the evaluation measures to be considered and presents a case study on the Kuwaiti currency notes. Section 7 discusses experiments performed and the results achieved, with an analysis and a discussion of the results, and Section 8 draws conclusions from experimental results and possible future work.

\section{Related Work}

To prevent counterfeiting, the security system of currency is encoded within the note, varying across different currencies. This persuaded researches in the area of currency recognition to emphasize discriminating real currency from counterfeit currency. In [5], the researchers studied banknotes from different countries as the main objective was to recognize currencies used in various countries. Features such as texture analysis, color analysis, and the size of the currency notes were used for currency recognition. A different study, also based on currency recognition, presented an approach to recognize serial numbers on the current Chinese currency notes [6]. This study used various components of image processing, including image binarization, morphological filtering, feature extraction, segmentation, and digit recognition. However, it did not focus on distinguishing between authentic and counterfeit currency notes.

In the literature, many researches have been developed when it comes to finding counterfeit banknotes. To verify Indian currency, the work in [7] used image processing techniques such as edge detection and image segmentation to compare between the output images of genuine and counterfeit banknotes. Their approach was used to extract characteristics of the Indian paper currency such as identification mark, security thread, and watermark. In [8], Canny operator was used for edge detection to capture and extract currency characteristics. The process of identifying original from counterfeit money in this paper was done by comparing the images after extracting important characteristics from both versions. The authors concluded that by using Canny operator for edge detection and image segmentation, the process of detecting original and counterfeit paper currency is considerably more rapid and effective. The proposed work in this paper also uses Canny operator because of its high efficiency in detecting edges, but differs from previous research by applying the operator to specific bit-planes instead of applying it to the original image.

With regards to applying bit-plane slicing technique to images, the work in [9] implemented this method on extracting features from an iris image. The paper observed that using canny operator for edge detection provided better results, but is computationally more expensive. The researchers used the fifth bit-plane for a better iris image to be able to extract features with Canny edge detection. 
Another paper used the same method as [9] for bacterial images, but used contouring method for detecting the edges of the images instead [10]. Different from the work proposed in [9], the authors here used the sixth and seventh planes for edge detection in bacterial images. Resultant edge images were compared with other edge detection methods and results showed that contouring method gave them the best results.

When it comes to recognizing counterfeit banknote images, no previous work was found on using bit-plane slicing technique with Canny edge detection method on currency images. As shown in the work of [9] and [10], feature extraction and image segmentation result in more accurate results when slicing images into bit-planes rather than using the actual image. In contrast to previous works, edges are detected from bit-planes using the Canny method for better results.

\section{Background}

\subsection{Counterfeit Currency}

The word 'currency' refers to money in any form when being used as a medium for exchange, especially circulating coins and banknotes. It represents an agreed medium both controlled and enforced by the state. The strength of societies with regards to trading with other societies depends highly on the value held by their currency [2]. Counterfeit money is currency created to imitate a government produced currency. Even though it is possible to counterfeit both paper and coin money, most of the counterfeit money today is paper, due to its higher value. Counterfeiting is as old as money itself, and is quite common throughout history, and has been referred to as the "world's second oldest profession" [11].

Towards the end of the twentieth century, advancements in the computer and photocopy industries enabled unskilled people to copy currency easily through the use of digital graphics. To catch up with these technological advances, new features had to be included in currency notes, such as color changing features, strips and micro printing. Some software programs, such as Adobe Photoshop, have been modified by their manufacturers to prohibit manipulation of banknotes scanned images.

The objective of counterfeiters is to produce realistic reproductions of the real banknotes by mastering known security features. In general, security features are categorized into features that can be detected by human senses and features that cannot be detected by human senses and which require some basic tools to be able to detect them, such as a magnifying glass. The third category consists of genuine features that result from the manufacturing process and the interaction of raw materials, which can only be detected by forensic examiners [2]. In general, the first category is usually easiest to counterfeit than the second and third categories [12]. Most of the security features are classified under features that can be detected by human senses, specifically sight and touch. An example of this is adding a raised print on both sides of a banknote, making them user-friendly for the visually impaired.

\subsection{Digital Image Processing}

The increase of computer and software use in recent years has made digital image processing a powerful tool. A wide variety of methods and algorithms have been developed to process images and explore new applications in this field. Digitization involves sampling of images and quantization of the sampled values. Digitally, an image is represented in terms of pixels and these pixels can be expressed further in terms of bits.

Digital image processing is "the use of computer algorithms to perform image processing on digital image" as defined by [13]. Its main objective is to enhance image appearance and automatic processing. It can also be used to restore deteriorated 
photographs and is becoming more common in the movie and electronic games industries [14].

\section{Problem Definition}

The circulation of counterfeit currency affects many economies by causing inflation and financial losses whenever traders and organizations are not reimbursed by banks for the counterfeit money they receive. Other negative effects counterfeiting has on society include reduction in the value of real money and inflation, where there is an unauthorized artificial increase in money supply [15]. Even though technology has greatly advanced in the last decade or so, overcoming the creation of counterfeit currency "remains a distant future" [2]. Currency examiners have to level up to the skills of counterfeiters who seem to be continuing to go down the road of counterfeit production. In order to control and minimize the flow of counterfeit currency in economies, software dealing with currency notes recognition is used [2].

In billing machines, the detection of counterfeit currency notes is one of the most critical tasks performed by the machines, where a robust, reliable, and high processing technique is required to automatically recognize counterfeit from authentic currency [16], [12]. As mentioned in the previous section, some security features in currency notes are visible and may be detected easily by the basic human senses, such as color and size of the notes. However, this method is limited by the fact that the quality of banknotes is deteriorates over time, and such features may not be detectable by then [17]. Other than wearing out and getting damaged, some currency notes have very complex designs that impose some degree of difficulty when processed with automatic currency recognition [17].

Motivated by the problems stated above, a simple and efficient approach is proposed in this paper to meet the desired speed and accuracy requirements in detecting counterfeit currency notes. The importance of identifying worn out or encoded security features is crucial and addressed by using Canny operator for detecting edges on bit-planes extracted from the original images.

\section{Methodology}

The digital analysis of two-dimensional images of currency is based on processing the image acquired, with the use of a computer. The method used in this paper is processed using MATLAB with image processing toolbox. The methodology implemented in this paper consists of the following stages:

Stage 1. Image acquired by scanner or digital camera.

Stage 2. Pre-process the image by converting the image to grayscale, smoothing the image using median filter, and adjusting the image's contrast.

Stage 3. Get eight bit-planes of the image.

Stage 4. Apply Canny operator for edge detection on higher order of bit-planes.

Stage 5. Image segmentation is performed on the image.

Stage 6. Extract features and label connected components for comparisons.

The flow of detecting authentic and counterfeit images is shown in the block diagram in Figure 1. 


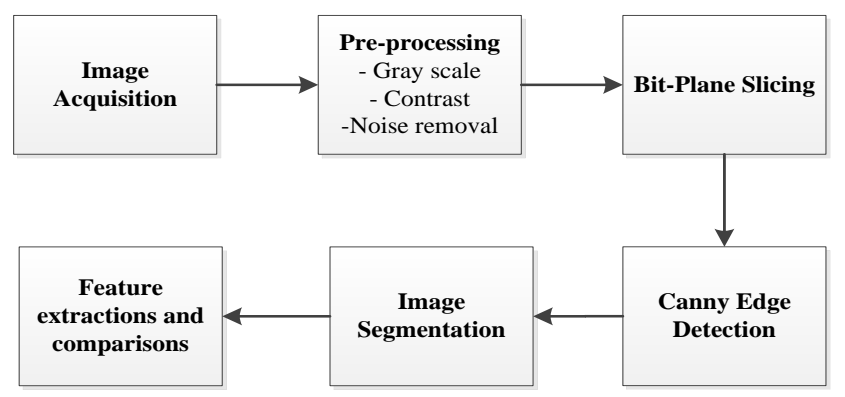

Figure 1. Block Diagram of Counterfeit Detection Process

\subsection{Image Acquisition}

This task is the most crucial phase in image processing. In order to obtain good results, a good quality of images is required to work with. Acquisition of images can be carried out in several ways, such as using a digital camera or scanner. In this work, a scanner is used to obtain images of genuine and counterfeit currency notes. The image format used is JPEG. Image acquisition should preserve all features existing in the actual version of the image.

\subsection{Pre-Processing}

This stage is concerned with the initial process applied on the raw images prior to image analysis and data extraction [17]. The main objective of pre-processing images is to enhance their visual appearance for more accurate results by converting the image to grayscale domain, smoothing and enhancing the image. The first task of pre-processing the currency note images is transforming the acquired image from RGB to grayscale domain, as it is quite difficult to deal with each of the color domains independently and perform tasks on them. Grayscale images are represented by pixels of shades of gray that range from the value of 0 (black) to 255 (white). It only shows the luminance information [4]. The work here is carried out on two dimensional matrices rather than the colored three dimensional matrices. This is obtained by the weighted sum of contribution by each component of the RGB domain as shown in equation 1 [18]:

$$
0.2989 \mathrm{R}+0.5870 \mathrm{G}+0.1140 \mathrm{~B}
$$

In the above equation, $\mathrm{R}$ stands for Red, $\mathrm{G}$ for Green, and $\mathrm{B}$ for Blue The weight associated with each component is not equal since green is the brightest, hence the highest weight. On the other hand, blue is the darkest hence holding the lowest weight [17]. Other than simplifying the work carried out on the images, grayscale images can save memory and computation time as well [9].

Scanned images are usually accompanied with noise. Image noise is the random variations of brightness in the images [15]. More specifically, it is the degradation in the image signal due to external disturbance that is automatically added at the time of capturing the image. At this stage of the proposed approach, to enhance image features, undesired noise has to be eliminated to be able to analyze and process the images further. Image smoothing is applied to remove noise from the images. This is performed using median filter. This filter was selected as it preserves the edges of an image and noise is reduced simultaneously. Median filter of a $3 \times 3$ neighborhood is used to compute the median, and pads the border of the input with 0's [3]. Figure 2 is an example of a median filter, where the center value in the $3 \times 3$ window is replaced by the median of all pixel values of that window. 


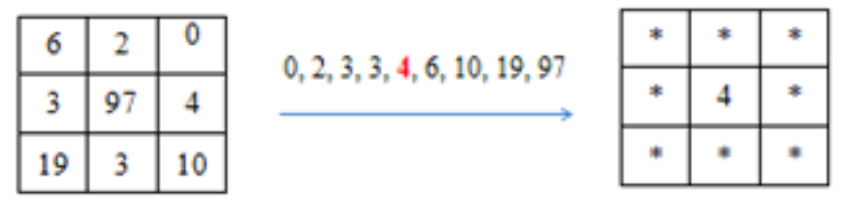

Figure 2. Example of $3 \times 3$ Median Filter

Image enhancement is the third task performed at this stage and is used to enhance images of low contrast. A histogram of a grayscale image represents the number of times each gray level is used in the image. By studying the histogram of a grayscale image, one can figure out that if gray level were clustered towards 0 , then it is a dark image. Brighter images will have the gray level clustered mostly at the upper end, towards the value of 255. An example demonstrates this in Figure 3. The sample currency image here is concentrated more towards the lighter and brighter values of the grayscale. Since the image is not dark, fewer pixels are located in the lower end of the grayscale.

In this scope of work, an entirely automatic procedure called histogram equalization is used to increase an image's contrast by evenly distributing its pixels [9]. However, enhancing images means fully brightening the pixels, which may not always be the desired method in some applications [4]. This drawback can be resolved by using a different contrast adjusting technique, such as imadjust. This MATLAB function works by distributing grayscale values along the entire range of the original image [3].

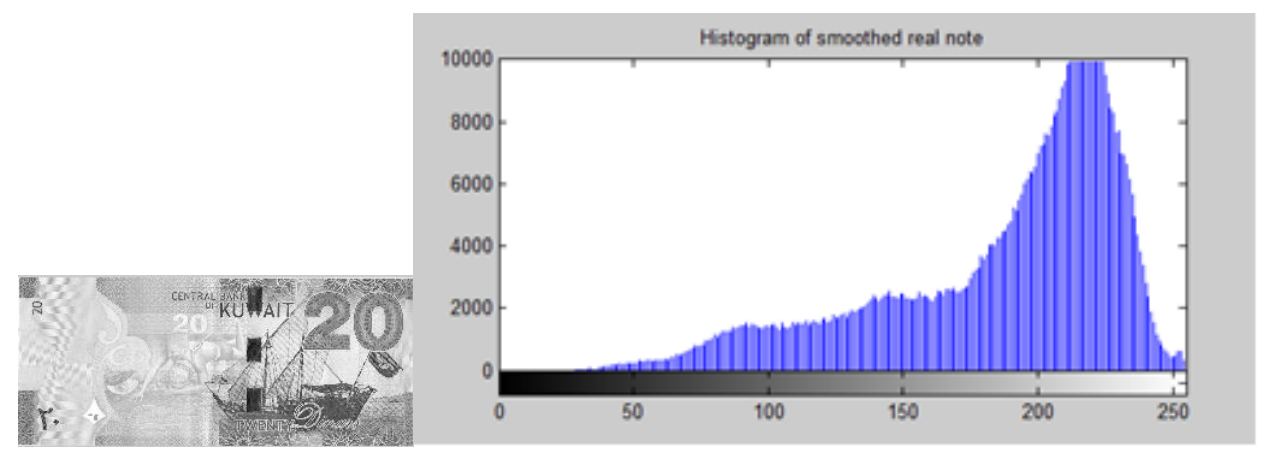

Figure 3. Histogram of a Sample Image

\subsection{Bit-Plane Slicing}

Bit-plane slicing technique is applied on the images of currency notes. The images, as suggested by the name, are "sliced" into different planes known as bitplanes [10]. Upon converting the colored images into grayscale images, these grayscale images can be transformed into a sequence of binary images by dividing them into their bit-planes. The intensity of each pixel has a value between 0 and 255, and each pixel in an image is represented by an 8-bit binary word [9]. For example, black pixels will be represented as 00000000, while white pixels are represented as 11111111 . This 8-bit grayscale image is composed of eight 1-bit planes. There may be a bit on each, some, or only one bit-plane depending on the intensity value of the pixel on the original image. If a colored image is separated into R, G, and B color components, each color component will have its own 8-bit plane. Thus, colored images will have a total of twenty four bit- planes.

As shown in Figure 4, bit-plane slicing technique ranges from plane 0, the Least Significant Bit (LSB), to plane 7, the Most Significant Bit (MSB). One major advantage of applying this technique is the retrieval of information contributed by 
each of the planes to the overall image. In most cases, the higher order bit-planes, fourth to seventh bit-planes, visualize most of the data of an image, while not a lot of information can be obtained for the lowest four bit-planes because of their lower contrast [4].

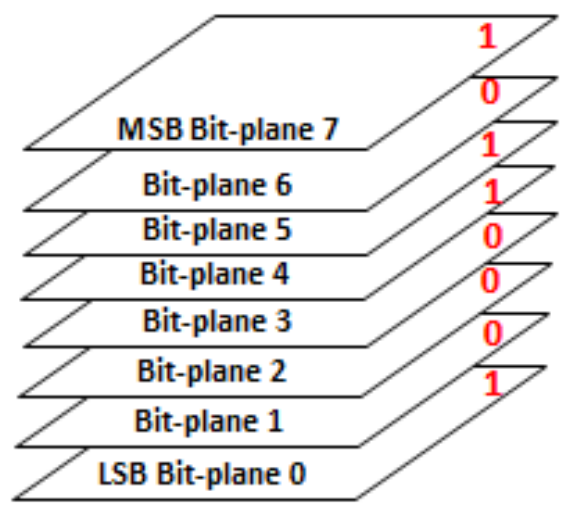

Figure 4. Slicing an Image into Eight Bit-Planes for 10110001

The bit-plane slicing technique can be used in many different ways. It is very efficient with image compression and enhancement of digital images. It can also be used to eliminate features, highlight them or emphasize some specific information. When applying the bit-plane slicing technique on images of a genuine and a counterfeit banknote, and then performing an edge detection method, the results in Figure 5 to 8 clearly display more valuable information in the higher bit-planes than lower bit-planes for both banknotes. As a result, most of the work in this research was carried out on the higher planes, more specifically bit-planes six and seven.

Furthermore, bit-plane slicing is known for saving storage space and saving space when sending information [14]. This technique is used in this paper to compute CPU time required to detect edges from a bit-plane and for faster feature extraction.

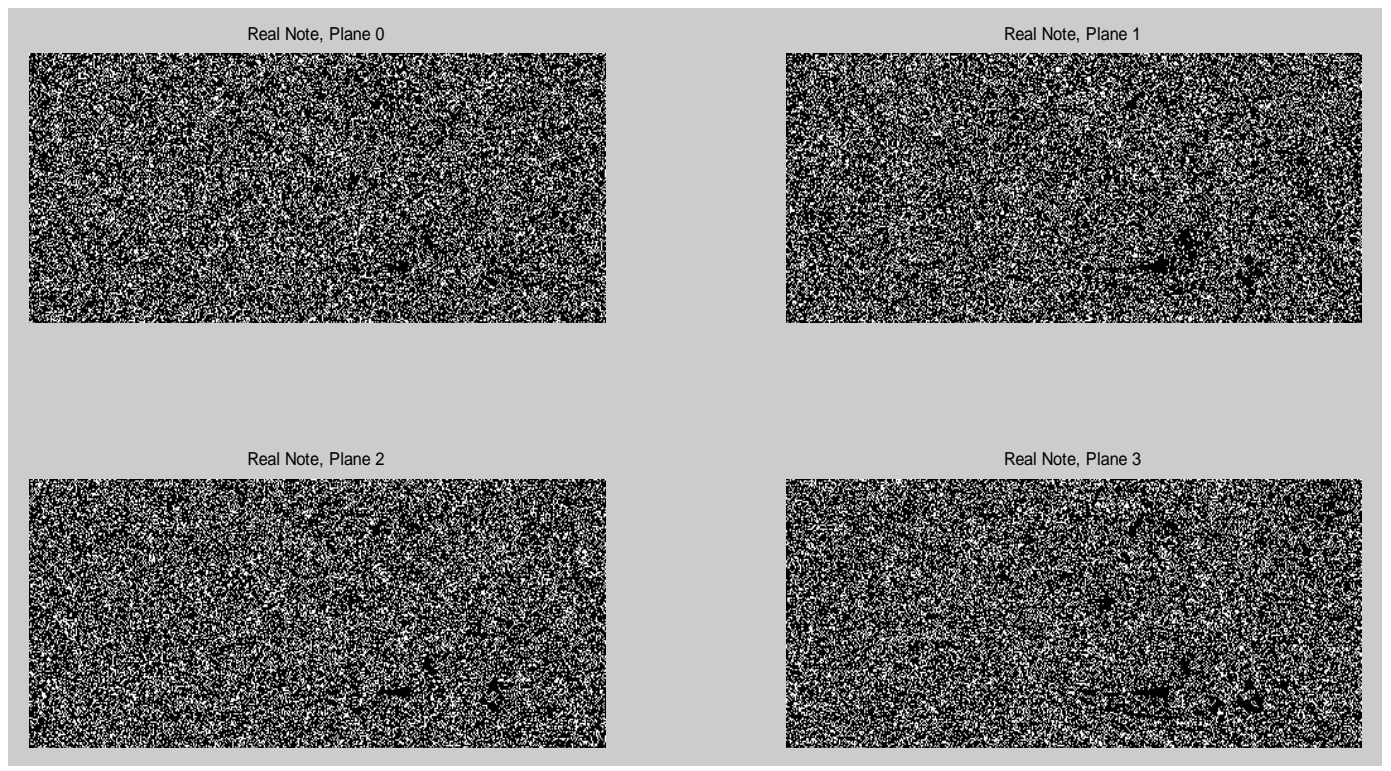

Figure 5. Canny Edge Detection Applied on Bit-Planes 0 to 3 (Left to Right) on a Genuine 20 KD Banknote 


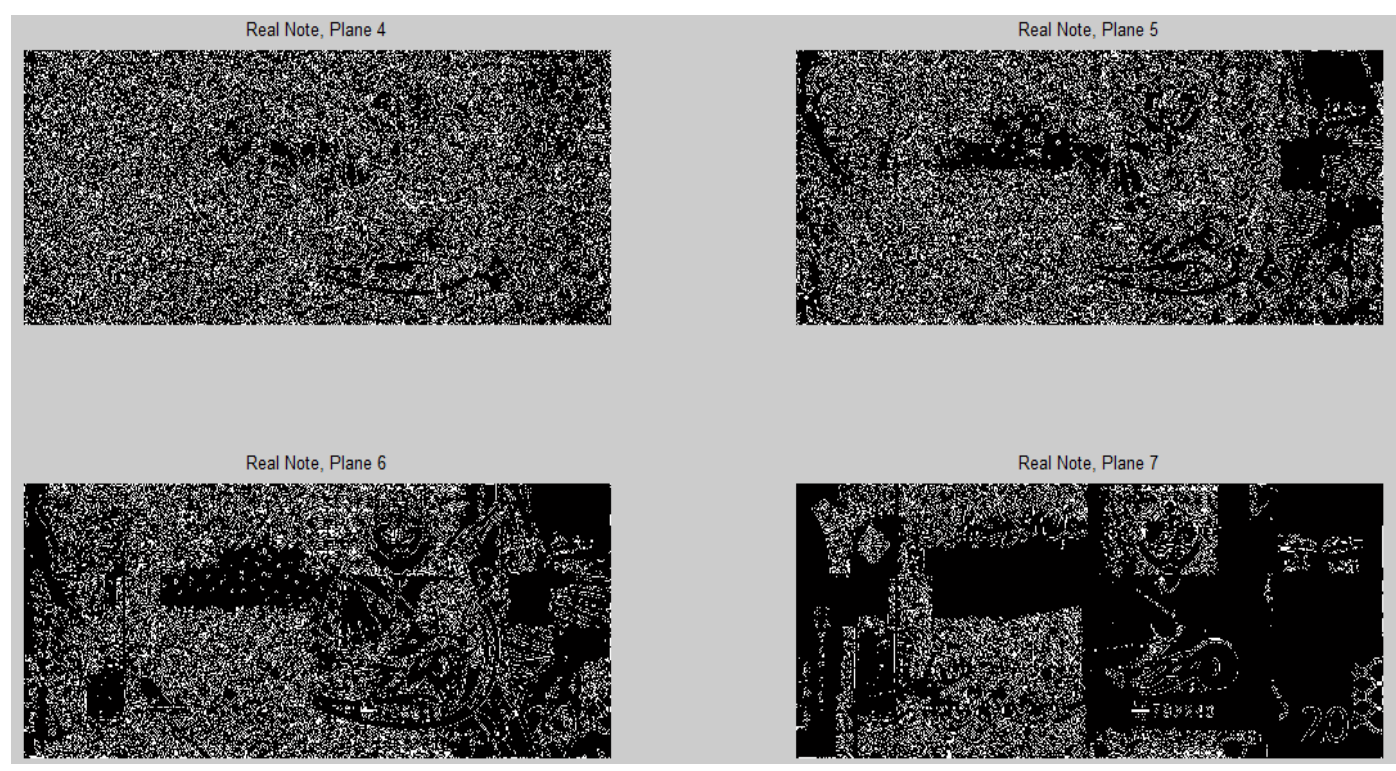

Figure 6. Canny Edge Detection Applied on Bit-Planes 4 to 7 (Left to Right) on a Genuine 20 KD Banknote

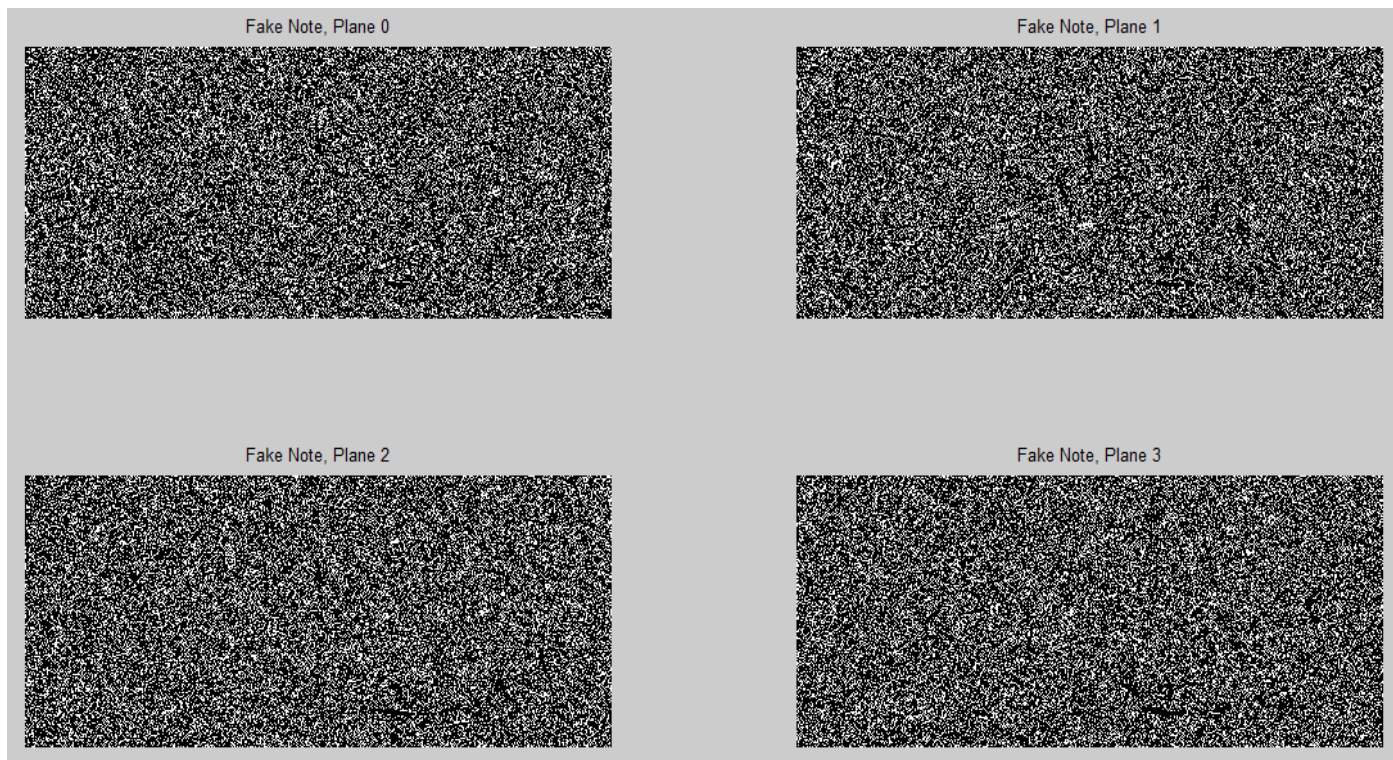

Figure 7. Canny Edge Detection Applied on Bit-Planes 0 to 3 (Left to Right) on a Counterfeit 20 KD Banknote 

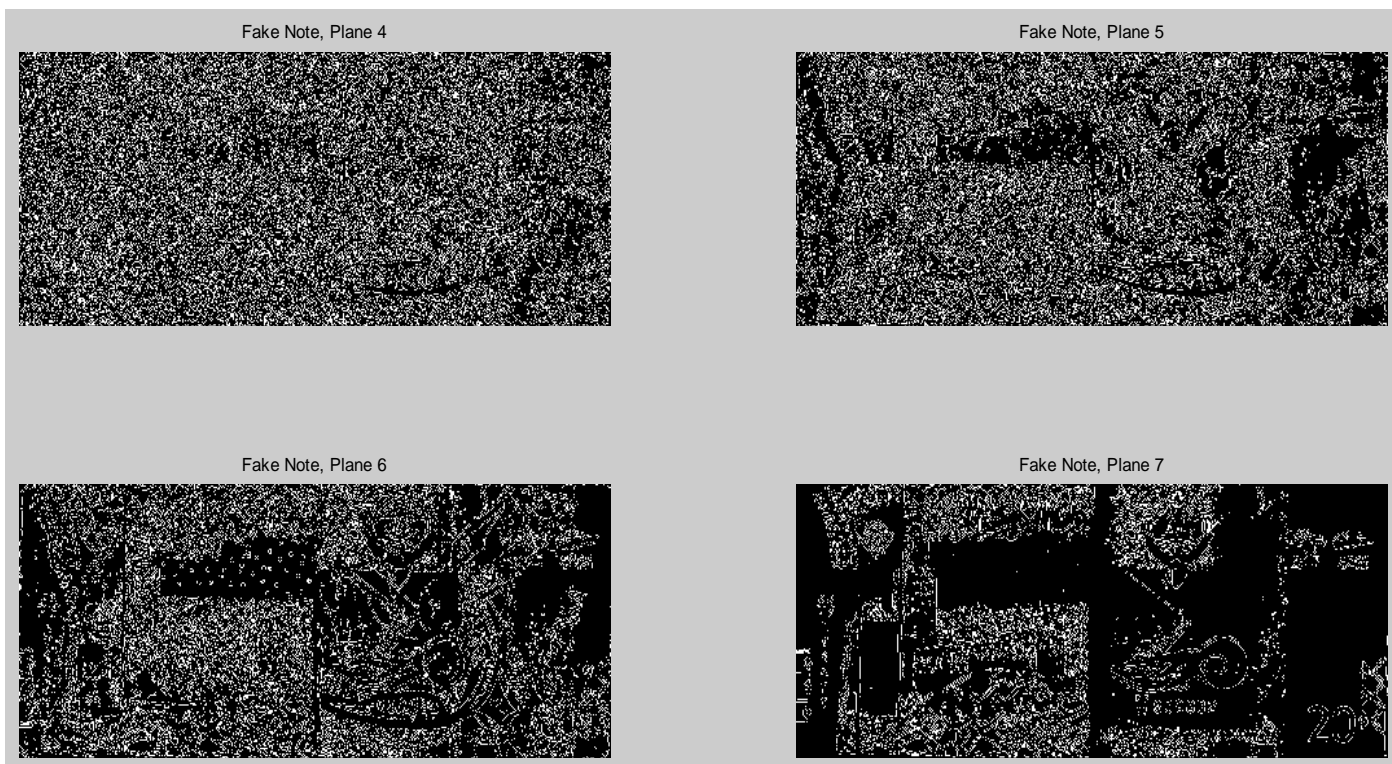

Figure 8 Canny Edge Detection Applied on Bit-Planes 4 to 7 (Left to Right) on a Counterfeit 20 KD Banknote

\subsection{Edge Detection}

One of the fundamental tools used in image processing and computer vision techniques is edge detection, specifically when features are required to be detected and extracted from an image. Edge detection algorithms are applied to images to identify object boundaries within an image. This is done by recognizing points of an image where brightness changes sharply, or where discontinuities exist in brightness [15]. Edge detection is mainly used for extracting information from an image and image segmentation [17].

Edge detecting algorithms used in the field of image processing include Canny, Laplacian of Gaussian, Sobel, Perwitt, and Roberts [23]. The Canny edge detector is known to be the most powerful of all edge detection methods where weaker edges can be detected without being misled by image noise [24]. The algorithm is able to find weak edges by marking points as edges if their amplitude is higher than their neighbors [2]. The basic idea behind this algorithm includes the following steps [22]:

Step 1: The raw image is smoothed by convolving it with a Gaussian filter. The equation of the Gaussian filter in two dimensions is represented as

$$
\mathrm{G}(\mathrm{x}, \mathrm{y})=\left(1 / 2 \pi \sigma^{2}\right) \mathrm{e}^{\wedge}-\left(\mathrm{x}^{2}+\mathrm{y}^{2} / 2 \sigma^{2}\right)
$$

Where $\sigma$ is the standard deviation of the Gaussian distribution, the distance from the origin in the horizontal direction is represented as $\mathrm{x}$, while $\mathrm{y}$ represents the distance in the vertical direction.

Step 2: Computing gradient magnitude and direction using edge detection operators, such as Sobel operator. Applying edge detection operators return first derivative values in the horizontal direction, $G_{x}$, and in the vertical direction, $G_{y}$. The gradient magnitude is then calculated using equation 3 and the gradient direction is calculated using equation 4 .

$$
\begin{aligned}
& |G|=\left(G_{x}{ }^{2}+G_{y}{ }^{2}\right)^{1 / 2} \\
\theta= & \arctan \left(G_{y} / G_{x}\right)
\end{aligned}
$$


Step 3: Non-maximum suppression is applied to eliminate pixels that do not compose the edge and only local maxima is marked as an edge. This is done by checking if a pixel is the local maximum amongst its neighborhood in the gradient direction obtained in the previous step. By removing pixels that do not compose the edges, a binary image with thinner edges is displayed as a result.

Step 4: Finally, hysteresis thresholding is used to detect strong edges with pixels of gradient magnitude higher than the upper threshold, while pixels of gradient values below the lower threshold are eliminated. Weak edges that are connected to strong edges are also detected using hysteresis thresholding.

After slicing the images into bit-planes, Canny edge detection is applied on each of the bit-planes. This is different than previous research in this area by analyzing bit-planes rather than analyzing the image itself. However, this is similar to the work applied on iris images in [9].

\subsection{Image Segmentation}

Image segmentation partitions a digital image into the required essential regions represented as multiple segments, a set of pixels [13]. It is basically used to detect and locate objects and boundaries, such as the lines and curves of an image. These segments collectively form the original image or a set of contours extracted from the image [13]. Image segmentation is basically used to simplify an image to offer an easier and more meaningful representation of the image for analyzing and extracting useful information [13].

For image segmentation, bwlabel operation is used to label the connected components in an image or part of an image. A connected component in a binary image is a set of pixels that form a connected group [3]. Each connected component is assigned a unique label to be able to visualize them in an image. The example in Figure 9 shows that the binary image has three connected components.

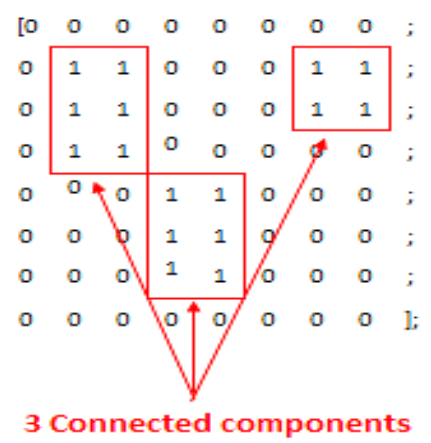

Figure 9. An Example of a Binarized Image with Three Connected
Components

\subsection{Feature Extraction}

The main purpose of analyzing images is the extraction of information beneficial for solving application problems. Feature extraction is a special form of an image that is reduced dimensionally. Specific visual content for indexing and retrieval can be captured in this phase [15]. This captured content is then used to perform the required task rather than working on the full size image where some information may be redundant and irrelevant. To ease the process of detecting edges of the captured image, binarization is used to convert gray level images into binary images that consist of only two colors, black and white [8]. Feature extraction undergoes the process of simplifying the data required to describe the larger set of data. This 
method is used in the scope of this work to identify visible and hidden security features in currency notes to be used for comparison between genuine and counterfeit images.

\section{Evaluation}

\subsection{Performance Metrics}

Resulting images will be critically evaluated to determine if the outputs of the proposed approach meet the requirements of being able to identify counterfeit currency notes by extracting features from the scanned images. The method proposed will also be compared to existing work of [8] where the same edge detection operator is used, but dissimilar in applying it only on the higher ordered bits of the obtained images.

\subsection{Experimental Setup}

The method used in this paper to detect counterfeit banknotes is processed on a Core i5 CPU with 4GB RAM using MATLAB 2013a along with image processing toolbox. This toolbox supports a wide range of image processing operations such as opening image file, adding noise to an image, adaptive filtering, etc. Sample images used to test proposed approach include a sixth issue authentic and counterfeit $20 \mathrm{KD}$ banknote, two fifth issue counterfeit $10 \mathrm{KD}$ banknotes, and one authentic fifth issue $10 \mathrm{KD}$ banknote. Authentic notes will be used as the reference images to base comparisons on.

\subsection{Case Study}

A case study on the Kuwaiti currency is used to evaluate the proposed work. The issuing of the Kuwaiti currency is solely exercised by the Central Bank of Kuwait (CBK), and is the state's exclusive privilege. The Kuwaiti currency unit is the Kuwaiti Dinar (KD) and notes are issued by the CBK in denominations of quarter Dinar, half Dinar, one Dinar, five Dinars, ten Dinars, and twenty Dinars. Since 1961, there have been a total of six issues of the Kuwaiti currency notes, with the most recent issue, the sixth issue, released into circulation on June 29, 2014 [20].

According to CBK, one can recognize the difference between real and counterfeited money in the $20 \mathrm{KD}$ denomination as per the following [20]:

Features 1 and 2: Raised print of the banknote's value on both sides of the banknote and the symbols can be felt. This feature has been added for the visually impaired.

Features 3 and 4: When held up to the light, the falcon watermark with the brighter value on each banknote can be seen and the incomplete shapes that combine to form the banknote's value.

Feature 5: The banknote can be tilted to see the wave shapes change color.

Feature 6: Circles in the solid art print can be seen when banknote is tilted.

Feature 7: The banknote's backside features a security thread that changes color upon tilting, as well as revealing artwork and the banknote's value upon directing light on.

Security features existing in the fifth issue of $10 \mathrm{KD}$ denominations are as per the below:

Feature 1: Hologram in the shape of a falcon head containing the value numerals of the note in Arabic can be seen when the banknote is titled.

Features 2 and 3: When held up to the light, a watermark of the second falcon head and the latent image of Arabic numeral value "10" in the water vessel can be seen. 
Feature 4: When held up to a light source, the security thread is viewed as a solid line rather than a series of disconnected vertical dashes.

Feature 5: Under an Ultraviolet light, this feature can be seen.

Security measures implemented by the CBK in the sixth issue denomination of 20 $\mathrm{KD}$ and fifth issue denomination of $10 \mathrm{KD}$ are shown in Figure 10 and Figure 11.

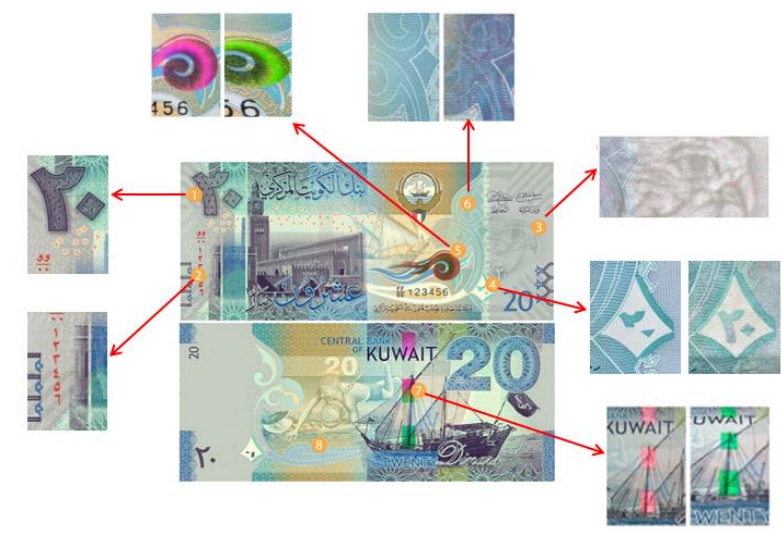

Figure 10. Security Features in Genuine Sixth Issue Denomination of 20 KD

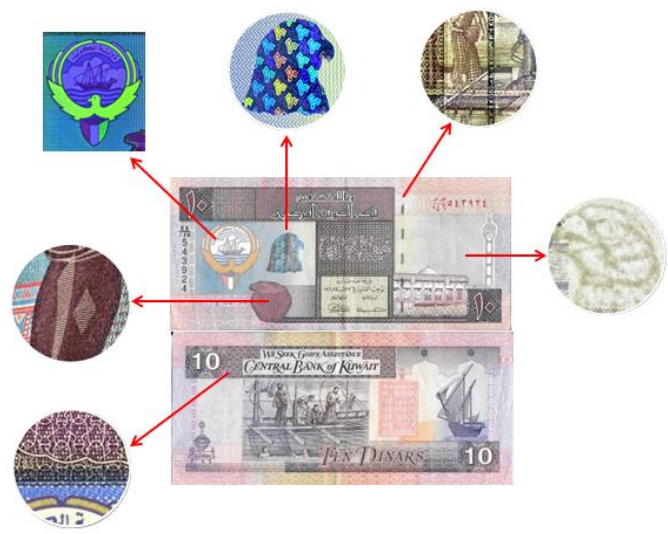

Figure 11. Security Features in Genuine Fifth Issue Denomination of 10 KD
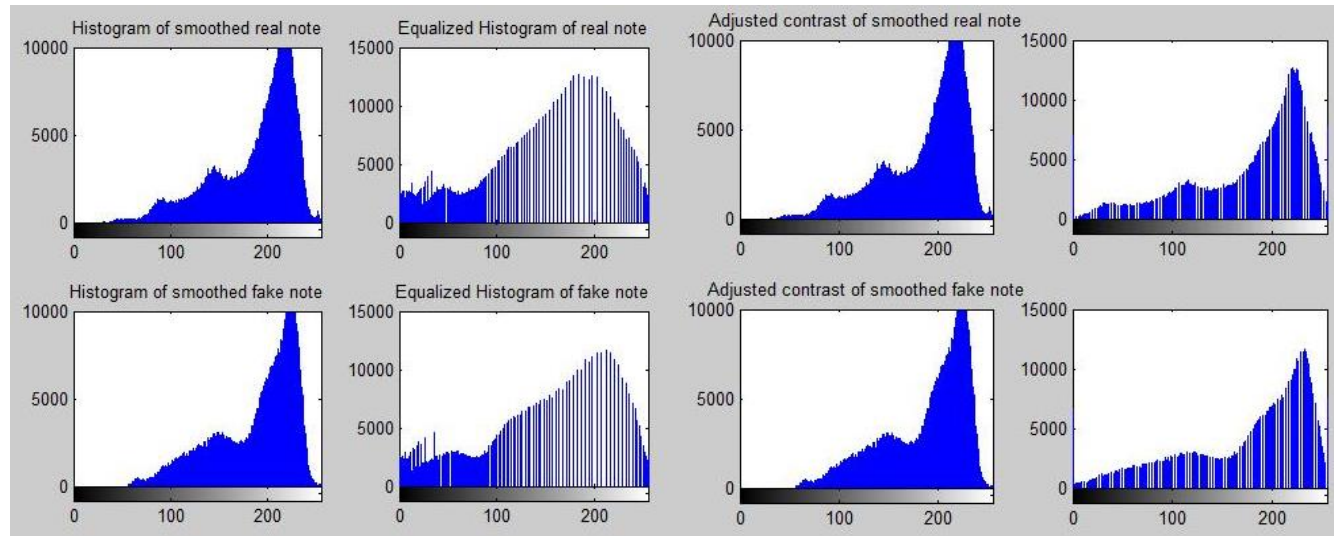

(a)

(b)

Figure 12. (a) Histogram and Equalized Histograms of a Genuine and Counterfeit Notes after Smoothing the Images. (b) Histogram and Equalized Histograms of a Genuine and Counterfeit Notes after Adjusting the Contrast of the Images 


\section{Discussion}

During the pre-processing stage, one may notice some differences between images of genuine and counterfeit notes. For instance, a comparison of histograms and equalized histograms of both notes after being smoothed to remove noise, and after being sharpened to enhance and highlight details within the images are shown in Figure 12 (a) and (b).

Several features were extracted from genuine and counterfeit Kuwaiti currency notes and compared in the number of components available in each note. If this number does not closely match, then the note is not genuine. Figure 13 (a) shows a feature that was extracted from a $20 \mathrm{KD}$ note and compared with its fake version (b). Figure 14 (a), (b) and (c) also show the difference between features extracted from a genuine $10 \mathrm{KD}$ and a counterfeit one. Another feature of the $10 \mathrm{KD}$ denominations was also extracted to discover the differences between an authentic and genuine note. The security thread in Figure 15 (a) appears clearly, whereas the thread is mostly undetectable in the counterfeit notes in Figure 15 (a) and (b). The position of the security thread also varies from the genuine note, implying that the notes are counterfeit.

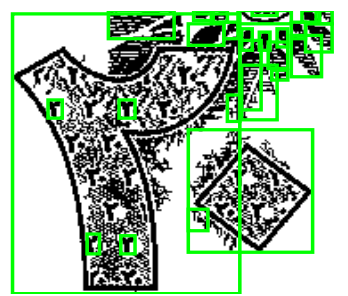

(a)

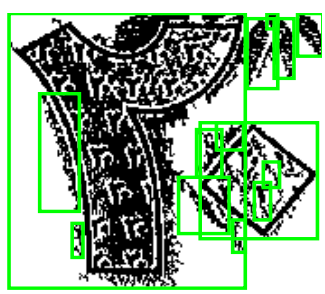

(b)

Figure 13. (a) Feature 1 Extracted from Genuine note. (b) The Same Feature Extracted from Counterfeit Note

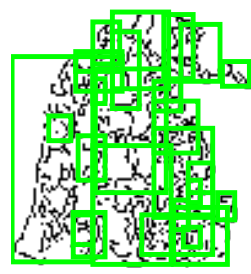

(a)

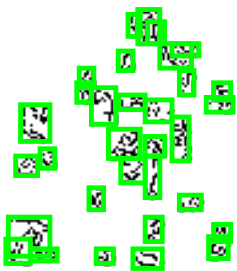

(b)

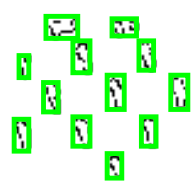

(c)

Figure 14. (a) Feature 1, Falcon Head, Extracted from Authentic Fifth Issue 10KD, (b) and (c) Falcon Head Extracted from Two different Counterfeit Fifth Issue 10KD

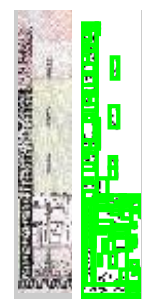

(a)

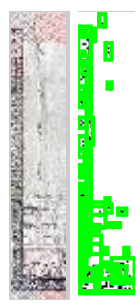

(b)

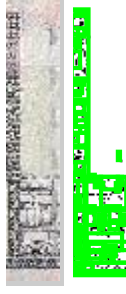

(c)

Figure 15. (a) Feature 4, Security Thread, Extracted from a Genuine 10 KD, (b) and (c) different Banknotes of the Same Denomination 
It is clearly shown that the genuine currency note allows extraction of features in a clear manner where the smaller ' 20 "'s can be seen. On the other hand, detailed features on the counterfeit note were not detectable, even though components with less than 30 pixels have been excluded from the images. The results obtained from comparing authentic and counterfeit banknotes are recorded in Table 1.

Table 1. Comparisons between Genuine and Counterfeit 20 KD Notes

\begin{tabular}{|l|l|l|}
\hline & $\begin{array}{l}\text { Genuine } \\
\text { Note }\end{array}$ & $\begin{array}{l}\text { Counterfeit } \\
\text { Note }\end{array}$ \\
\hline $\begin{array}{l}\text { Connected } \\
\text { components }\end{array}$ & & \\
\hline Overal & 444.00 & 367.00 \\
\hline Feature 1 & 27.00 & 14.00 \\
\hline Mean values & & \\
\hline Grayscale - Original & 185.20 & 186.20 \\
\hline Grayscale-6th bit-plane & 0.71 & 0.73 \\
\hline Red channel & 172.56 & 179.80 \\
\hline Green channel & 189.64 & 188.69 \\
\hline Blue channel & 195.68 & 190.25 \\
\hline Standard Deviation & & \\
\hline Original image & 51.05 & 49.35 \\
\hline 6th bit-plane & 0.45 & 0.44 \\
\hline SNR & & \\
\hline Original image & 3.62 & 3.77 \\
\hline 6th bit-plane & 1.58 & 1.65 \\
\hline
\end{tabular}

SNR is the signal-to-noise-ratio and is measured in decibels (dB). SNR is a measure that compares the level of the desired signal to the level of background noise. It is obtained from equation 5 [4].

$$
\mathrm{SNR}=\text { Mean } / \text { Standard Deviation }
$$

Other than extracting features from both authentic and fake currency notes, the images were compared against the output they produced when applying Canny edge detection. Authentic notes showed more information than those fake notes as shown in Figure 16 (a) and (b).

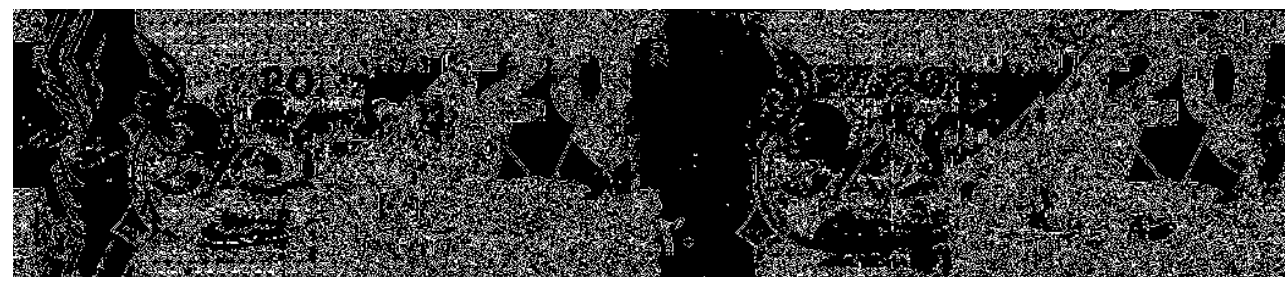

(a)

(b)

Figure 16. (a) (Top) Canny Edge Detection on the Sixth Plane of the Genuine Note. (b) (Bottom) Canny Edge Detection on the Sixth Plane of the Fake Note

In the work of [8], Canny operator for edge detection was applied on currency images to identify genuine notes from forged ones. However, the method proposed in this paper shows that there is significant improvement in CPU time performance when running the proposed method for Canny edge detector on a single bit-plane rather than running it on the original image without being sliced. For both forged 10 
KD notes, the seventh bit-plane contained most of the image data and hence was used to compare the execution performance of using Canny operator on the actual image. Results are shown in Figure 17. The blue plots in Figure 17 represent results from comparing an authentic image of a fifth issue $10 \mathrm{KD}$ with a counterfeit one. The red plots show the same comparison, but with a different fifth issue counterfeit $10 \mathrm{KD}$ image. It is quite obvious from the graph in Figure 17 that the proposed approach using Canny operator for detecting edges of an image's higher bit-planes provides faster results than using this method directly on the actual image. The same comparison was performed on a sixth issue $20 \mathrm{KD}$ and results are shown in Figure 18.

Unfortunately, paper currency can be easily damaged by tears or wearing out in different areas of the note, thus adding to the complexity of currency inspection. Currency notes in warm climates tend to wear faster than those in cooler climates. Such factors are abused by counterfeiters by intentionally damaging the forged notes and covering up suspicious areas of the notes [2].

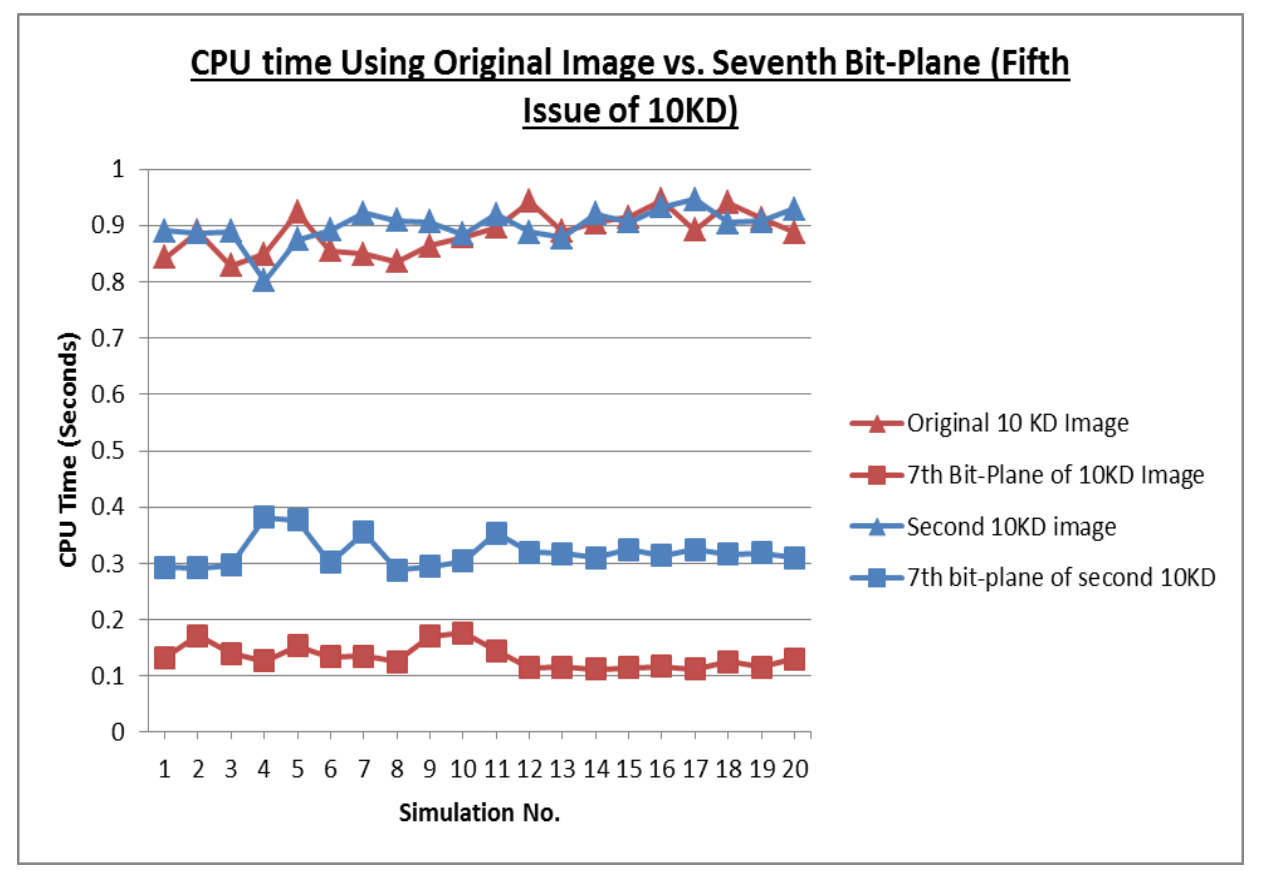

Figure 17. CPU Time Comparison between Algorithms of Using Canny Operator for Detecting Edges in a Counterfeit $10 \mathrm{KD}$ Image and Using the Same Edge Detecting Operator on the Seventh Plane of the Counterfeit 10 KD Image 


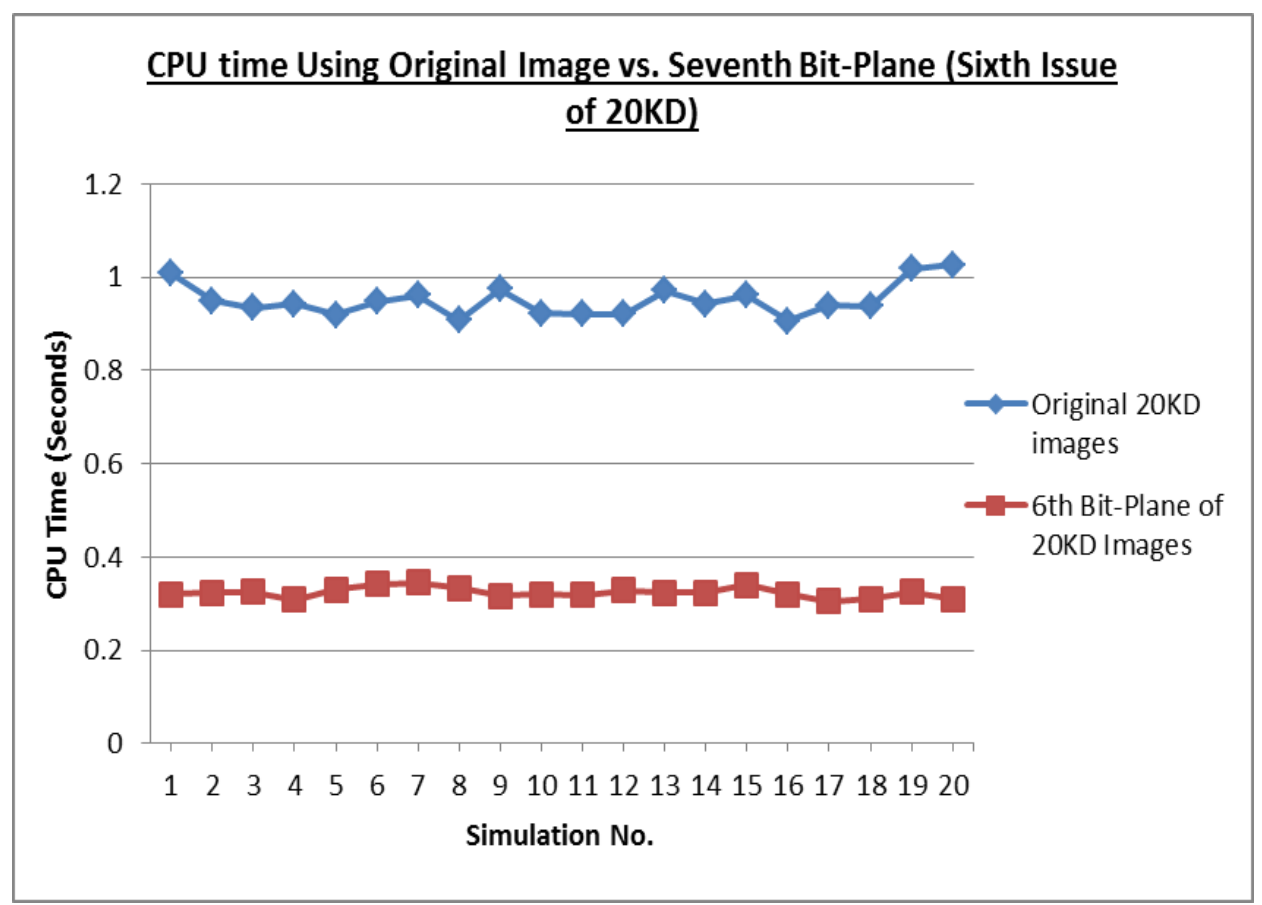

Figure 18. CPU Time Comparison between Algorithms of Using Canny Operator for Detecting Edges in a Counterfeit $20 \mathrm{KD}$ Image and Using the same Edge Detecting Operator on the Seventh Plane of the Counterfeit 20 KD Image

\section{Conclusion and Future Work}

In this paper, the most powerful and commonly used edge detection technique, Canny operator, was studied and applied on all the eight bit-planes. It was observed that the sixth and seventh bit-plane provide better results in recognizing edges and hidden features of images. Canny operator was proved to provide the most significant results when applied on both of those bit-planes in the grayscale image. It was also proved that this edge detection algorithm has a low error rate and has a good localization of edges where the computed edges are not far from the actual edges of an image, unlike the other algorithms. Canny operator managed to find all strong edges and those weak edges connected to strong edges without being fooled by image noise. Experimental results show that applying edge detection algorithms on one of the higher bit-planes performed at a much faster rate than executing the algorithm on the actual image. Furthermore, security features in images of genuine and counterfeit Kuwaiti currency notes were extracted and compared using image segmentation and Canny operator to identify those counterfeited notes.

As proposed by [4], future work can include extending this work to using sixth bit-plane with colored images to verify if using colored bit-planes would yield more details than grayscale bit-planes.

\section{References}

[1] “MP Submits Query On Bringing In Banknotes”, Arab Time, (2015), April 20.

[2] J. Chambers, W. Yan, A. Garhwal and M. Kankanhalli, "Currency security and forensics: a survey", Multimedia Tools and Applications, vol. 74, no. 11, (2015), pp. 4013-4043.

[3] R. C. Gonzalez and R. E. Woods, "Digital Image Processing (3rd Edition)", Prentice-Hall, Inc., Upper Saddle River, NJ, USA, (2006).

[4] M. Sathik and N. Parveen, "Feature Extraction on Colored X-ray Images by Bit-Plane Slicing Technique", International Journal of Engineering Science and Technology, vol. 2, no. 7, (2010), pp. 2820-2824. 
[5] H. Hassanpour, A. Yaseri and G. Ardeshiri, "Feature Extraction for Paper Currency Recognition". Proceedings of the 9th International Symposium on Signal Processing and Its Applications (ISSPA), Sharjah, United Arab Emirate, December 12-15, (2007).

[6] J. Qian, D. Qian and M. Zhang, "A Digit Recognition System for Paper Currency Identification Based on Virtual Instruments", International Conference on Information and Automation, China, December 15-17, (2006).

[7] R. Mirza and V. Nanda, "Paper Currency Verification System Based on Characteristic Extraction Using Image Processing", International Journal of Engineering and Advanced Technology (IJEAT), vol. 1, no. 3, February (2012), pp. 68-71.

[8] M. Akbar, A. Sedayu, A. Putra and S. Widyarto, "Original and Counterfeit Money Detection Based on Edge Detection", Information Technology and Biomedical Engineering, Indonesia, November (2013).

[9] K. Khobragade and K. Kale, "Iris Edge Detection with Bit-Plane Slicing Technique", International Journal of Engineering Science and Technology, (2014), pp. 27-31.

[10] P. Kalavathi, "An Efficient Edge Detection Method Based on Bit-plane Slicing for Bacterial Images," Oriental Journal of Computer Science \& Technology, India, vol. 6, no. 3, (2013), pp. 315-320.

[11] http://en.wikipedia.org/wiki/Counterfeit_money, accessed October, 10, (2015).

[12] S. Ali1, M. Gogoi and S. Mukherjee, "Challenges in Indian Currency Denomination Recognition \& Authentication", International Journal of Research in Engineering and Technology, vol. 3, no. 11, November (2013).

[13] M. Deborah and C. Prathap, "Detection of Fake Currency using Image Processing", International Journal of Innovative Science, Engineering \& Technology, vol. 1, no. 10, December (2014).

[14] N. Hernandez and J. Quirarte, "Bits Planes Technique for Digital Image Processing", Electrical Engineering, Computing Science and Automatic Control, 2008, November (2008), pp. 186-191.

[15] M. Thakur and A. Kaur, "Various Fake Currency Detection Techniques", International Journal of Engineering Science and Technology, vol. 1, no.11, July (2014), pp. 1309-1313.

[16] S. Battiato, G. M. Farinella, A. Bruna and G. C. Guarnera, "Counterfeit Detection and Value Recognition of Euro Banknotes”, Sensors, vol. 13, no. 2, (2013), pp. 2515-2529.

[17] R. Mirza and V. Nanda, "Design and Implementation of Indian Paper Currency Authentication System Based on Feature Extraction by Edge Based Segmentation Using Sobel Operator", International Journal of Engineering Research and Development, vol. 2, no. 2, August (2012), pp. 41-46.

[18] http://www.mathworks.com/help/matlab/ref/rgb2gray.html, accessed October, 10, (2015).

[19] J. Patel, M. Jain and P. Dutta, "Fault Detection Using Graph Based Segmentation", International Journal of Engineering Science and Technology, vol. 2, no. 11, November (2013), pp. 2845-2851.

[20] "The Central Bank of Kuwait", Available online http://www.cbk.gov.kw/en/images/leaflet-10-1149513.pdf, accessed October 10, (2015).

[21] http://www.mathworks.com/help/matlab/ref/rgb2gray.html, accessed October, 10, (2015).

[22] S. S. Al-Amri, N. V. Kalyankar and S. D. Khamitkar. "Image Segmentation by Using Edge Detection", International Journal on Computer Science and Engineering, vol. 2, no. 3, (2010), pp. 804-807.

[23] Z. Xiaofeng and X. Hongsheng, "A Study on Denoising Method for Image of Mixed Noise", International Journal of Multimedia and Ubiquitous Engineering (IJMUE), vol. 9, no. 8, (2014), pp. 419-428.

[24] Y. C. Hu, B. H. Su, W. L. Chen and W. Y. Lu, "Image Zooming for Indexed Color Images Based on Bilinear Interpolation", International Journal of Multimedia and Ubiquitous Engineering (IJMUE), vol. 7, no. 2, (2012), pp 353-358.

\section{Authors}

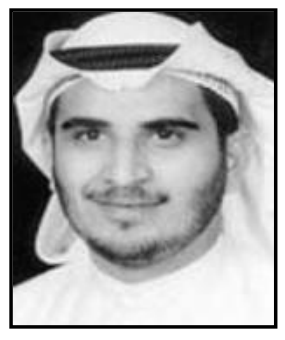

Mohammad H. Alshayeji, received his B.Sc. (Computer Engineering) from the University of Miami and his MS (Computer Science) from the University of Central Florida. He got his Ph.D. (Computer Science) from University of Southern California. Currently he is working as Assistant Professor in Computer Engineering Department, College of Computing Sciences and Engineering, Kuwait University.

Dr. Alshayeji has several publications in different international Journals and Conferences. His research interests include Multimedia, Image Processing, Cloud Computing, Security and Distributed Systems.3 


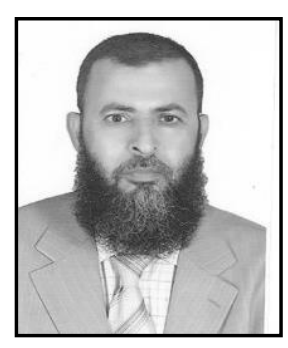

Mohammad Al-Rousan, received his BSc in Computer Engineering from King Saud University, Saudi Arabia, in 1986. $\mathrm{He}$ received his M.S. in Electrical and Computer Engineering from University of Missouri-Columbia, MI, USA in 1992. In 1996, he was awarded the $\mathrm{PhD}$ in Electrical and Computer Engineering from Brigham Young University, UT, USA. Currently AL-Rousan is a full professor at the Department of Computer engineering, Kuwait University. He is on sabbatical leave from Jordan University of Science and Technology, Jordan. His technical interests include AI systems, Image processing, and Nano Technology.

Dunya T Hassoun, holds a BSc in Computer Engineering (2009) and is currently pursuing MSc in Computer Engineering from the College of Computing Sciences and Engineering, Kuwait University. She is a Microsoft Certified IT Professional and works as a Systems Specialist at a private college in Kuwait. Her research interests include image processing and data mining. 